\title{
Tensor coupling and pseudospin symmetry in nuclei
}

\author{
P. Alberto \\ Departamento de Física and Centro de Física Computacional, Universidade de Coimbra, P-3004-516 Coimbra, Portugal \\ R. Lisboa ${ }^{1}$ and M. Malheiro ${ }^{1,2}$ \\ ${ }^{1}$ Instituto de Física, Universidade Federal Fluminense, 24210-340 Niterói, Brazil \\ ${ }^{2}$ Departamento de Física, Instituto Tecnológico de Aeronáutica, CTA, 12228-900, São José dos Campos, Brazil \\ A. S. de Castro \\ Departamento de Física e Química, Universidade Estadual Paulista, 12516-410 Guaratinguetá, SP, Brazil
}

(Received 30 November 2004; published 22 March 2005)

\begin{abstract}
In this work we study the contribution of the isoscalar tensor coupling to the realization of pseudospin symmetry in nuclei. Using realistic values for the tensor coupling strength, we show that this coupling reduces noticeably the pseudospin splittings, especially for single-particle levels near the Fermi surface. By using an energy decomposition of the pseudospin energy splittings, we show that the changes in these splittings come mainly through the changes induced in the lower radial wave function for the low-lying pseudospin partners and through changes in the expectation value of the pseudospin-orbit coupling term for surface partners. This allows us to confirm the conclusion already reached in previous studies, namely that the pseudospin symmetry in nuclei is of a dynamical nature.
\end{abstract}

DOI: 10.1103/PhysRevC.71.034313

PACS number(s): 21.10.Hw, 21.30.Fe, 21.60.Cs

\section{INTRODUCTION}

Pseudospin symmetry is a concept that appeared in nuclear physics more than 30 years ago $[1,2]$ to account for the observation, in heavy nuclei, of the quasidegeneracy of orbitals with quantum numbers $(n, l, j=l+1 / 2)$ and $(n-1, l+2, j=l+3 / 2)$ (for fixed $n$ and $l$ ). Such pairs of single-particle states are known as pseudospin partners. This doublet structure is related to the pseudo-orbital angular momentum and pseudospin quantum numbers $\tilde{l}=l+1$ and $\tilde{s}=s=1 / 2$ [3-7], respectively. The former, as noted by Ginocchio [7], is just the orbital angular momentum of the lower component of the Dirac spinor. Pseudospin partners are doublets with the same $\tilde{l}$. For example, for the partners $\left[n s_{1 / 2},(n-1) d_{3 / 2}\right], \tilde{l}=1$, and for $\left[n p_{3 / 2},(n-1) f_{5 / 2}\right]$ one has $\tilde{l}=2$.

The existence of degenerate or quasidegenerate pseudospin partners is connected to a $\mathrm{SU}(2)$ symmetry of the Dirac equation with only scalar $S$ and vector $V$ potentials such that $V=-S$, regardless of the particular shapes of these potentials [8]. It happens that in the relativistic mean-field theories of nuclei (RMF) [9-11], the sum $\Sigma=S+V$ is small at the nuclear energy scale, so this symmetry, known as pseudospin symmetry, provides a natural explanation of the existence of quasidegenerate pseudospin partners in nuclei referred to previously [7,12-14]. However, because in RMF theories $\Sigma$ acts as binding potential for the nucleons, it is not possible to have exact pseudospin symmetry in nuclei. Further works have shown that the particular shape of $\Sigma$, not just its smallness, can affect the pseudospin energy splittings and also explain the isospin dependence of these splittings [14-17]. Moreover, using an energy decomposition coming from the Schrödinger-like equation for the lower component of the Dirac spinor, it was shown that the observed pseudospin splitting arises from a cancellation of the several energy components and not primarily from the pseudospin-orbit term, which is proportional to the derivative of $\Sigma[15,16]$. Altogether, this led us to conclude that, in nuclei, pseudospin symmetry is realized in a dynamical way. A similar conclusion was reached by Marcos et al. [18,19].

The tensor coupling has been used in studies of nuclear properties with effective Lagrangians, including RMF theories by Furnstahl et al. in Ref. [20], and in the relativistic Hartree approach model, studied by Mao in Ref. [21]. Those works assessed its influence on nuclear observables, namely the spin-orbit splitting of single-particle levels in nuclei, the result being that the tensor coupling, a higher order term in a relativistic expansion, increases significantly the spin-orbit coupling. This suggests that the tensor coupling could have a significant contribution to pseudospin splittings in nuclei as well. This contribution is expected to be particularly relevant for the levels near the Fermi surface, because the tensor coupling depends on the derivative of a vector potential, which has a peak near the Fermi surface for typical nuclear mean-field vector potentials.

The tensor coupling has also been used as a natural way to introduce the harmonic oscillator in a relativistic (Dirac) formalism. In a recent article, it was shown that the harmonic oscillator with scalar and vector potentials can exhibit an exact pseudospin symmetry [22,23]. When this symmetry is broken $(\Sigma \neq 0)$, the breaking term is quite large, manifesting its nonperturbative behavior. However, if a tensor coupling is introduced, the form of harmonic-oscillator potential can still be maintained with $\Sigma=0$, but the pseudospin symmetry is broken perturbatively [24].

The tensor interaction has also been considered to explain how the spin-orbit term can be small for $\Lambda$-nucleus and large in the nucleon-nucleus case [25]. It is assumed that in the 
strange sector (case of $\Lambda$ ) the tensor coupling is large and the spin-orbit term obtained from this interaction can cancel in part the contribution coming from the scalar and vector interactions. This result shows that the tensor interaction can change strongly the spin-orbit term. In this spirit, we want to investigate if this interaction can also affect the pseudospinorbit term (spin-orbit of the lower component) $[5,6,15]$. We address this problem by performing a mean-field calculation for the neutron levels of ${ }^{208} \mathrm{~Pb}$, using mean-field Lorentz vector and scalar potentials with a Woods-Saxon shape. These potentials were used in the previous works that revealed the dynamical nature of pseudospin symmetry. In this work we will perform a similar calculation, including now a tensor coupling term, and using again an energy decomposition similar to the one used in [15]. We find that the tensor coupling potential has a noticeable effect on the pseudospin splittings. We study in particular detail the changes of the radial wave functions for the pseudospin partners and the differences between low-lying and near-the-Fermi-surface pseudospin doublets.

This article is organized as follows. In Sec. II A we present the Lagrangian for Fermion fields coupled to external scalar, vector, and tensor fields and obtain the corresponding singleparticle Dirac Hamiltonian. The Dirac equations of motion are obtained in Sec. II B, with emphasis on the second-order differential equations for the upper and lower components of the Dirac spinor. In Sec. II C we perform the energy decomposition based on the second-order differential equation for the lower component of the spinor, which will allow us to analyze the contribution of the radial tensor potential $U$ for the pseudospin energy splittings. The results of the calculation using mean-fields with Woods-Saxon shape for $S$ and $V$ radial potentials (thereby also fixing the tensor potential) are presented in Sec. III, together with a discussion of the effects of the tensor potential on the neutron pseudospin partners, both for deep levels and levels near the Fermi surface. The wave functions for the radial lower components of those levels are also plotted and their influence on the pseudospin splittings are discussed. Finally, our conclusions are summarized in Sec. IV.

\section{DIRAC EQUATION WITH ISOSCALAR TENSOR COUPLING}

\section{A. Dirac Hamiltonian}

Using the conventions of G. Mao [21], the nucleon-meson Lagrangian density of a nuclear mean-field theory with nucleons interacting with $\sigma, \omega$, and $\rho$ mesons, in which a tensor (derivative) coupling is included, reads $(\hbar=c=1)$ as follows:

$$
\begin{aligned}
\mathcal{L}= & \bar{\Psi}\left(i \gamma^{\mu} \partial_{\mu}-M\right) \Psi-g_{\sigma} \bar{\Psi} \sigma \Psi-g_{\omega} \bar{\Psi} \gamma_{\mu} \Psi \omega^{\mu}-g_{\rho} \bar{\Psi} \gamma_{\mu} \\
& \times \frac{\vec{\tau}}{2} \cdot \Psi \vec{\rho}^{\mu}-\frac{f_{\omega}}{4 M} \bar{\Psi} \sigma^{\mu \nu} \Psi \omega_{\mu \nu}-\frac{f_{\rho}}{4 M} \bar{\Psi} \sigma^{\mu \nu} \frac{\vec{\tau}}{2} \cdot \Psi \vec{\rho}_{\mu \nu}
\end{aligned}
$$

In this Lagrangian, $\omega_{\mu \nu}=\partial_{\mu} \omega_{\nu}-\partial_{\nu} \omega_{\mu}, \vec{\rho}_{\mu \nu}=\partial_{\mu} \vec{\rho}_{\nu}-$ $\partial_{\nu} \vec{\rho}_{\mu}$, and $\sigma^{\mu \nu}=i / 2\left[\gamma^{\mu}, \gamma^{\nu}\right]$. Here the only vector mesons we are going to consider are the isoscalar $\omega$ mesons. Furthermore, in a mean-field theory, the meson fields are static and only the timelike component is considered, that is, we have the following:

$$
\sigma=\sigma(\boldsymbol{r}) \quad \omega_{\mu}=\omega(\boldsymbol{r}) \delta_{\mu 0} .
$$

The Hamiltonian density is then given by the following:

$$
\begin{aligned}
\mathcal{H}= & \frac{\partial \mathcal{L}}{\partial\left(\partial_{0} \Psi\right)} \partial_{0} \Psi-\mathcal{L}=\bar{\Psi} \gamma^{0} \partial_{0} \Psi-\mathcal{L}=-i \Psi^{\dagger} \boldsymbol{\alpha} \cdot \nabla \Psi \\
& +\bar{\Psi} M \Psi+g_{\sigma} \bar{\Psi} \sigma \Psi+g_{\omega} \Psi^{\dagger} \Psi \omega-i \frac{f_{\omega}}{2 M} \Psi^{\dagger} \beta \boldsymbol{\alpha} \cdot \nabla \omega \Psi .
\end{aligned}
$$

The corresponding single-particle (Dirac) Hamiltonian is as follows:

$$
H=-i \boldsymbol{\alpha} \cdot \nabla+\beta\left(M+g_{\sigma} \sigma\right)+g_{\omega} \omega-i \frac{f_{\omega}}{2 M} \beta \boldsymbol{\alpha} \cdot \nabla \omega .
$$

If we now define the scalar $S$ and vector $V$ potentials as, respectively, $S=g_{\sigma} \sigma$ and $V=g_{\omega} \omega$, this last equation reads as follows:

$$
H=-i \boldsymbol{\alpha} \cdot \nabla+\beta(M+S)+V-i \beta \boldsymbol{\alpha} \cdot \mathcal{U},
$$

where $\mathcal{U}=f_{\omega} /(2 M) \nabla \omega$. Moreover, if the field $\omega(\boldsymbol{r})$ is just a function of the radial coordinate $r$, this Hamiltonian becomes the following:

$$
H=-i \boldsymbol{\alpha} \cdot \nabla+\beta(M+S)+V-i \beta \boldsymbol{\alpha} \cdot \hat{\boldsymbol{r}} U,
$$

where $U$ is the radial function as follows:

$$
U(r)=\frac{f_{\omega}}{2 M} \omega^{\prime}=\frac{1}{2 M} \frac{f_{\omega}}{g_{\omega}} V^{\prime}
$$

In the remainder of the article we use the notation $f_{v} \equiv f_{\omega} / g_{\omega}$ for the sake of simplicity.

\section{B. Equations of motion}

The Dirac equation for nucleons with tensor coupling is written as follows:

$$
H \Psi=\mathcal{E} \Psi,
$$

where $H$ is given by Eq. (4).

It is instructive to decompose this equation into two secondorder equations for the upper and lower components of the spinor $\Psi$ but retaining their spinor structure. To this end, we use the projectors $P_{ \pm}=(I \pm \beta) / 2$ applied to $\Psi$, that is, define the spinors $\Psi_{ \pm}=P_{ \pm} \Psi$. Applying $P_{ \pm}$to the left of the Dirac equation (6) we obtain the following:

$$
\begin{gathered}
\boldsymbol{\alpha} \cdot \boldsymbol{p} \Psi_{-}+(M+S+V) \Psi_{+}-i \boldsymbol{\alpha} \cdot \hat{\boldsymbol{r}} U \Psi_{-}=\mathcal{E} \Psi_{+}, \\
\boldsymbol{\alpha} \cdot \boldsymbol{p} \Psi_{+}+(-M-S+V) \Psi_{-}+i \boldsymbol{\alpha} \cdot \hat{\boldsymbol{r}} U \Psi_{+}=\mathcal{E} \Psi_{-},
\end{gathered}
$$

or, defining $\Sigma=V+S, \Delta=V-S$, and $E=\mathcal{E}-M$,

$$
\begin{gathered}
(\boldsymbol{\alpha} \cdot \boldsymbol{p}-i \boldsymbol{\alpha} \cdot \hat{\boldsymbol{r}} U) \Psi_{-}=(E-\Sigma) \Psi_{+}, \\
(\boldsymbol{\alpha} \cdot \boldsymbol{p}+i \boldsymbol{\alpha} \cdot \hat{\boldsymbol{r}} U) \Psi_{+}=(E+2 M-\Delta) \Psi_{-} .
\end{gathered}
$$

Using the formulas in the Appendix we finally obtain the following:

$$
\begin{aligned}
p^{2} \Psi_{-} & +\left(U^{2}-U^{\prime}-2 \frac{U}{r}-\frac{\Sigma^{\prime} U}{E-\Sigma}\right) \Psi_{-} \\
& -\frac{\Sigma^{\prime}}{E-\Sigma} \frac{\partial \Psi_{-}}{\partial r}+\left(-4 U+2 \frac{\Sigma^{\prime}}{E-\Sigma}\right) \frac{\boldsymbol{L} \cdot \boldsymbol{S}}{r} \Psi_{-} \\
= & (E-\Sigma)(E+2 M-\Delta) \Psi_{-},
\end{aligned}
$$




$$
\begin{aligned}
\boldsymbol{p}^{2} \Psi_{+} & +\left(U^{2}+U^{\prime}+2 \frac{U}{r}+\frac{\Delta^{\prime} U}{E+2 M-\Delta}\right) \Psi_{+} \\
& -\frac{\Delta^{\prime}}{E+2 M-\Delta} \frac{\partial \Psi_{+}}{\partial r}+\left(4 U+2 \frac{\Delta^{\prime}}{E+2 M-\Delta}\right) \\
& \times \frac{\boldsymbol{L} \cdot \boldsymbol{S}}{r} \Psi_{+}=(E-\Sigma)(E+2 M-\Delta) \Psi_{+} \cdot
\end{aligned}
$$

The terms with $\boldsymbol{L} \cdot \boldsymbol{S}$ for the upper and lower components are spin-orbit and pseudospin orbit coupling terms, respectively. We can note immediately that the tensor potential contributes to both. A full analysis is done in the next section.

If $S, V$, and $U$ are radial functions, then the general solution of Eq. (6) is as follows:

$$
\Psi_{\kappa m}(\boldsymbol{r})=\left(\begin{array}{l}
i \frac{g_{\kappa}(r)}{r} \mathcal{Y}_{\kappa m}(\hat{\boldsymbol{r}}) \\
\frac{f_{\kappa}(r)}{r} \mathcal{Y}_{-\kappa m}(\hat{\boldsymbol{r}})
\end{array}\right) .
$$

Here $\kappa$ is the quantum number related to the total angular momentum $j$ and orbital momentum $l$ by the following:

$$
\kappa=\left\{\begin{array}{clrl}
-(l+1) & =-(j+1 / 2), & & j=l+1 / 2, \\
l & =+(j+1 / 2), & & j=l-1 / 2 .
\end{array}\right.
$$

The spinor spherical harmonics $\mathcal{Y}_{\kappa m}$ result from the coupling of the two-dimensional spinors to the eigenstates of orbital angular momentum and form a complete orthonormal set. Through the following relations:

$$
\begin{aligned}
& j=|\kappa|-\frac{1}{2}, \\
& \ell=|\kappa|+\frac{1}{2}\left(\frac{\kappa}{|\kappa|}-1\right),
\end{aligned}
$$

one sees that if the upper component of the spinor in Eq. (13) has a orbital quantum number $l$, the lower component (which has quantum number $-\kappa$ ) must have a orbital angular momentum $\tilde{l}=l-\kappa /|\kappa|$. This quantum number has been associated with the pseudospin symmetry [7].

Using the property $\boldsymbol{\sigma} \cdot \hat{\boldsymbol{r}} \mathcal{Y}_{\kappa m}=-\mathcal{Y}_{-\kappa m}$, Eqs. (9) and (10) reduce to a set of two coupled first-order ordinary differential equations for the radial upper and lower components $g_{\kappa}$ and $f_{\kappa}$, namely

$$
\begin{aligned}
& {\left[\frac{d}{d r}+\frac{\kappa}{r}-U(r)\right] g_{\kappa}(r)=[E+2 M-\Delta(r)] f_{\kappa}(r),} \\
& {\left[\frac{d}{d r}-\frac{\kappa}{r}+U(r)\right] f_{\kappa}(r)=-[E-\Sigma(r)] g_{\kappa}(r) .}
\end{aligned}
$$

Similarly, from Eqs. (11) and (12) we arrive at the following second-order differential equations for $g_{\kappa}$ and $f_{\kappa}$ as follows:

$$
\begin{aligned}
\left\{\frac{d^{2}}{d r^{2}}-\right. & \frac{\kappa(\kappa+1)}{r^{2}}+\frac{\Delta^{\prime}}{E+2 M-\Delta(r)}\left[\frac{d}{d r}+\frac{\kappa}{r}-U(r)\right] \\
& \left.+2 \kappa \frac{U(r)}{r}-U^{\prime}(r)-U^{2}(r)\right\} g_{\kappa}(r) \\
= & -[E-\Sigma(r)][E+2 M-\Delta(r)] g_{\kappa}(r)
\end{aligned}
$$

$$
\begin{aligned}
\left\{\frac{d^{2}}{d r^{2}}-\right. & \frac{\kappa(\kappa-1)}{r^{2}}+\frac{\Sigma^{\prime}}{E-\Sigma(r)}\left[\frac{d}{d r}-\frac{\kappa}{r}+U(r)\right] \\
& \left.+2 \kappa \frac{U(r)}{r}+U^{\prime}(r)-U^{2}(r)\right\} f_{\kappa}(r) \\
= & -[E-\Sigma(r)][E+2 M-\Delta(r)] f_{\kappa}(r) .
\end{aligned}
$$

These two equations show explicitly the new terms that depend on $U(r)$ and are originated by the tensor interaction. In particular, the term $2 \kappa U(r) / r$, which is the same for the upper and lower component, is the modification in the spin-orbit and pseudospin-orbit terms, respectively, generated by the tensor interaction.

\section{Energy decomposition and sum rule}

The terms in Eq. (11) with denominator $E-\Sigma$, which have a singularity in $E=\Sigma$, fulfill a sum rule coming from Eq. (A2). If one divides each member of that equation by $2 M^{*}=E+2 M-\Delta$, left multiply them by $\Psi_{-}^{\dagger}$ and integrate, one obtains the following:

$$
\begin{aligned}
\int \Psi_{-}^{\dagger} & \frac{i \boldsymbol{\alpha} \cdot \hat{\boldsymbol{r}}}{2 M^{*}} \Sigma^{\prime} \Psi_{+} d^{3} \boldsymbol{r} \\
= & P \int \Psi_{-}^{\dagger} \frac{\Sigma^{\prime}}{E-\Sigma} \frac{1}{2 M^{*}} \frac{\partial \Psi_{-}}{\partial r} \Psi_{-} d^{3} \boldsymbol{r} \\
& +P \int \Psi_{-}^{\dagger} \frac{\Sigma^{\prime}}{E-\Sigma} \frac{U}{2 M^{*}} \Psi_{-} d^{3} \boldsymbol{r} \\
& -P \int \Psi_{-}^{\dagger} \frac{\Sigma^{\prime}}{E-\Sigma} \frac{1}{M^{*}} \frac{\boldsymbol{L} \cdot \boldsymbol{S}}{r} \Psi_{-} d^{3} \boldsymbol{r}
\end{aligned}
$$

where $P$ stands for the principal value of the integral. In terms of the radial functions $g_{\kappa}$ and $f_{\kappa}$ the sum rule reads as follows:

$$
\begin{aligned}
& -\int_{0}^{\infty} f_{\kappa} \frac{\Sigma^{\prime}}{2 M^{*}} g_{\kappa} d r=P \int_{0}^{\infty} \frac{\Sigma^{\prime}}{E-\Sigma} \frac{1}{2 M^{*}} f_{\kappa}\left(\frac{f_{\kappa}}{r}\right)^{\prime} r d r \\
& \quad+P \int_{0}^{\infty} \frac{\Sigma^{\prime}}{E-\Sigma} \frac{U}{2 M^{*}} f_{\kappa}^{2} d r+P \int_{0}^{\infty} \frac{\Sigma^{\prime}}{E-\Sigma} \frac{1-\kappa}{2 M^{*}} f_{\kappa}^{2} d r .
\end{aligned}
$$

This sum rule can be used to check the numerical results.

The energy decomposition of Eq. (11) can be performed by dividing it by $2 M^{*}$ and computing its expectation value for the spinor $\Psi_{-}$, yielding the following:

$$
\left\langle\frac{p^{2}}{2 M^{*}}\right\rangle+\left\langle V_{U}\right\rangle+\left\langle V_{\Sigma^{\prime} U}\right\rangle+\left\langle V_{\text {Darwin }}\right\rangle+\left\langle V_{\mathrm{PSO}}\right\rangle+\langle\Sigma\rangle=E,
$$

$$
\begin{aligned}
V_{U} & =\frac{1}{2 M^{*}}\left(U^{2}-U^{\prime}-2 \frac{U}{r}\right), \\
V_{\Sigma^{\prime} U} & =-\frac{1}{2 M^{*}} \frac{\Sigma^{\prime} U}{E-\Sigma}, \\
V_{\text {Darwin }} & =-\frac{1}{2 M^{*}} \frac{\Sigma^{\prime}}{E-\Sigma} \frac{\partial}{\partial r},
\end{aligned}
$$




$$
\begin{aligned}
V_{\mathrm{PSO}} & =\frac{1}{M^{*}}\left(-2 U+\frac{\Sigma^{\prime}}{E-\Sigma}\right) \frac{\boldsymbol{L} \cdot \boldsymbol{S}}{r}, \\
\langle\mathcal{O}\rangle & \equiv \frac{\int \Psi_{-}^{\dagger} \mathcal{O} \Psi_{-} d^{3} \boldsymbol{r}}{\int \Psi_{-}^{\dagger} \Psi_{-} d^{3} \boldsymbol{r}} .
\end{aligned}
$$

For the terms with $E-\Sigma$ in the denominator the integral is taken in the principal value sense.

\section{TENSOR COUPLING WITH MEAN-FIELD WOODS-SAXON POTENTIALS}

As stated above, the aim of this article is to study the effect of the tensor coupling on the pseudospin splitting in nuclei. In previous works, in which we studied pseudospin symmetry in nuclei [14], we solved numerically the Dirac equation with central mean-field potentials with Woods-Saxon shapes. Although these potentials are not full self-consistent relativistic potentials derived from meson fields, they are realistic enough to be applied to many nuclei. In this article we follow the same approach, namely we consider the sum and difference potentials $\Sigma$ and $\Delta$ to be of the following general form:

$$
P(r)=\frac{P_{0}}{1+\exp [(r-R) / a]},
$$

whereas the tensor potential $U(r)$ is obtained by the following:

$$
U(r)=\frac{f_{v}}{2 M} V^{\prime}=\frac{f_{v}}{2 M} \frac{\Sigma^{\prime}+\Delta^{\prime}}{2} .
$$

The depth, $P_{0}$, the radius (range), $R$, and the diffusivity, $a$, for $\Sigma$ and $\Delta$ are fitted to reproduce the single-particle spectrum of ${ }^{208} \mathrm{~Pb}[14,15]$.

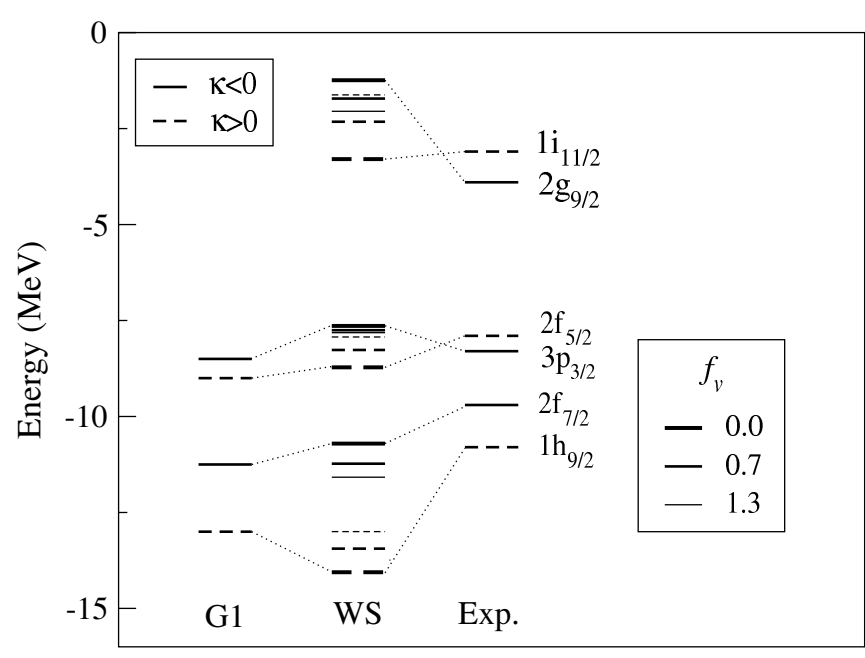

FIG. 1. Calculated neutron single-particle energy levels of the pseudospin partners $\left[2 f_{7 / 2}-1 h_{9 / 2}\right],\left[2 f_{5 / 2}-3 p_{3 / 2}\right]$, and $\left[1 i_{11 / 2}-\right.$ $\left.2 g_{9 / 2}\right]$ in ${ }^{208} \mathrm{~Pb}$. The left-most values are the corresponding values of the model G1 of Furnsthal et al. [26] and the experimental values [27] are in the right-most column. In the middle column (WS) is our calculation with Woods-Saxon potentials for three values of the tensor coupling strength $f_{v}$, corresponding to lines of different thickness. Pseudospin-partner levels with $\kappa<0$ are represented by solid lines, whereas those with $\kappa>0$ have dashed lines. The Woods-Saxon parameters used to fit ${ }^{208} \mathrm{~Pb}$ neutron energy levels are $R=7 \mathrm{fm}$, $\Delta_{0}=650 \mathrm{MeV}, \Sigma_{0}=-66 \mathrm{MeV}$, and $a=0.6 \mathrm{fm}$.

Using the general Woods-Saxon form in Eq. (25) for $\Sigma$ and $\Delta$, and with $U$ given by Eq. (26), we solved numerically the coupled first-order Dirac equations [Eqs. (17) and (18)]. The single-particle energy levels are shown in Fig. 1, when $f_{v}$ varies from 0 to 1.3. This range of values is consistent with

TABLE I. Values of energies and terms in the decomposition (23) for the pseudospin partners $\left[2 s_{1 / 2}-\right.$ $\left.1 d_{3 / 2}\right]$, [2 $\left.p_{3 / 2}-1 f_{5 / 2}\right],\left[3 p_{3 / 2}-2 f_{5 / 2}\right]$, and $\left[2 g_{9 / 2}-1 i_{11 / 2}\right]$ for two values of $f_{v}$. The energies and expectation values are given in $\mathrm{MeV}$.

\begin{tabular}{lcccrrrrr}
\hline \hline Partners & $f_{v}$ & $\left\langle p^{2} / 2 M^{*}\right\rangle$ & $\left\langle V_{U}\right\rangle$ & $\left\langle V_{\Sigma^{\prime} U}\right\rangle$ & $\left\langle V_{\text {Darwin }}\right\rangle$ & \multicolumn{1}{c}{$\left\langle V_{\mathrm{PSO}}\right\rangle$} & \multicolumn{1}{c}{$\langle\Sigma\rangle$} & $E$ \\
\hline \multirow{2}{*}{$s_{1 / 2}$} & 0.0 & 24.4396 & 0.0000 & 0.0000 & 3.9527 & -0.5852 & -61.4644 & -41.5627 \\
& 1.3 & 23.9037 & 0.2351 & -0.0943 & -3.6870 & -0.5632 & -61.9114 & -42.1170 \\
$1 d_{3 / 2}$ & 0.0 & 21.1032 & 0.0000 & 0.0000 & -0.8106 & 0.0966 & -64.4159 & -44.0266 \\
& 1.3 & 20.7075 & 0.1459 & -0.0712 & -0.4170 & 0.1559 & -64.6181 & -44.1170 \\
$2 p_{3 / 2}$ & 0.0 & 33.2950 & 0.0000 & 0.0000 & -2.7538 & -1.6340 & -60.2687 & -31.3615 \\
& 1.3 & 32.7195 & 0.2465 & 0.1223 & -2.7319 & -1.5835 & -60.8762 & -32.1033 \\
$1 f_{5 / 2}$ & 0.0 & 28.5114 & 0.0000 & 0.0000 & 0.7303 & 0.5384 & -64.5165 & -34.7365 \\
& 1.3 & 28.2218 & 0.2598 & -0.0021 & 0.9522 & 0.6780 & -64.6667 & -34.5571 \\
$3 p_{3 / 2}$ & 0.0 & 52.9135 & 0.0000 & 0.0000 & 0.8482 & -1.6013 & -59.8025 & -7.6420 \\
& 1.3 & 52.6913 & 0.5000 & 0.1669 & 0.9638 & -1.9231 & -60.2278 & -7.8280 \\
$2 f_{5 / 2}$ & 0.0 & 50.1727 & 0.0000 & 0.0000 & 2.3696 & 1.0254 & -62.2874 & -8.7197 \\
& 1.3 & 50.0072 & 0.6256 & 0.1427 & 2.3831 & 1.3089 & -62.3270 & -7.8595 \\
$2 g_{9 / 2}$ & 0.0 & 55.7666 & 0.0000 & 0.0000 & 2.8295 & -6.4555 & -53.3816 & -1.2410 \\
& 1.3 & 56.0706 & 0.5622 & 0.2855 & 2.6487 & -7.2110 & -54.4705 & -2.1140 \\
$1 i_{11 / 2}$ & 0.0 & 51.2033 & 0.0000 & 0.0000 & 3.1788 & 3.3530 & -61.0308 & -3.2958 \\
& 1.3 & 51.6221 & 0.8997 & 0.1529 & 2.9268 & 4.1391 & -61.2232 & -1.4826 \\
\hline \hline
\end{tabular}


that found in Ref. [20] for fittings of RMF and point coupling models to nucleon observables.

One sees clearly from Fig. 1 that turning on the tensor coupling decreases the pseudospin splittings of the levels near the Fermi surface. For $f_{v}=1.3$ the pair $\left[2 f_{5 / 2}-3 p_{3 / 2}\right]$ becomes almost degenerate, whereas the $\left[1 i_{11 / 2}-2 g_{9 / 2}\right]$ doublet even reverses its order. Interestingly, the experimental energy values for these two pairs show this order reversal, which is not reproduced by the model calculations without tensor coupling. In Fig. 1, when we increase the tensor coupling $f_{v}$, the energy for pseudospin partners with $\kappa<0$ become deeper and those with $\kappa>0$ become more unbounded. This systematics indicates that pseudospin symmetry is improved by the tensor interaction.

To better understand why and how tensor coupling affects pseudospin splittings, we computed the contributions from the terms of the energy decomposition in Eq. (23) to the energy splittings for both low-lying and close-to-the-Fermi-surface pseudospin partners.

In Table I we can see what is the contribution of all the terms of that energy decomposition for the two lowest neutron pseudospin partners ([2s $\left.s_{1 / 2}-1 d_{3 / 2}\right]$ and $\left[2 p_{3 / 2}-\right.$ $\left.\left.1 f_{5 / 2}\right]\right)$ and for the two top-most neutron pseudospin partners ([3 $\left.p_{3 / 2}-2 f_{5 / 2}\right]$ and $\left.\left[2 g_{9 / 2}-1 i_{11 / 2}\right]\right)$ for two values of the tensor coupling strength: $f_{v}=0.0$ (no tensor coupling) and $f_{v}=1.3$. As expected, because tensor interaction is a higher order interaction in the Lagrangian (derivative term) scaled by $1 / M$, the changes in the energy produced by the potential terms $V_{U}$ and $V_{\Sigma^{\prime} U}$ are small in comparison with the kinetic and potential terms, as shown in Table I. This table also shows that these terms, together with the pseudospin-orbit term, are significantly bigger for the surface levels than for the lower levels. This agrees with our expectations, referred before, that the effect of the tensor coupling is larger for the surface levels, because the potential $U$ is proportional to the derivative of the vector potential. Conversely, changes induced by the tensor coupling in $\left\langle V_{\Sigma^{\prime} U}\right\rangle$ and $\left\langle V_{\text {Darwin }}\right\rangle$ for surface levels are smaller.

The smallness of the terms containing the tensor potential in regard to the kinetic and $\Sigma$ potential terms is misleading concerning the effect in the pseudospin symmetry, because the changes of these last terms with $f_{v}$ are small, whereas the corresponding changes in $\left\langle V_{\mathrm{PSO}}\right\rangle$ and $\left\langle V_{U}\right\rangle$ can be significant for the surface levels, especially when compared with the energies of these levels. Note that the values of $\left\langle V_{\mathrm{PSO}}\right\rangle$ can have quite different values for different levels. This is because of the respective values of the $\kappa$ quantum number, as explained below.

To have a better understanding of the influence of all these terms in the pseudospin splittings, we plot in Figs. 2(a) and (b) the splittings and the differences of the terms in Eq. (23) for the pseudospin doublets $\left[2 s_{1 / 2}-1 d_{3 / 2}\right]$ and $\left[2 g_{9 / 2}-1 i_{11 / 2}\right]$ as a function of $f_{v}$. From these figures is clear that the decrease of the pseudospin energy splitting $\Delta E$ is much more pronounced for the surface doublet than for the low-lying one and would be even more if we considered the relative energy variations.

A more detailed analysis of the several contributions to the pseudospin energy splittings reveals that for the deep pseudospin doublet the contribution of the pseudospin-orbit
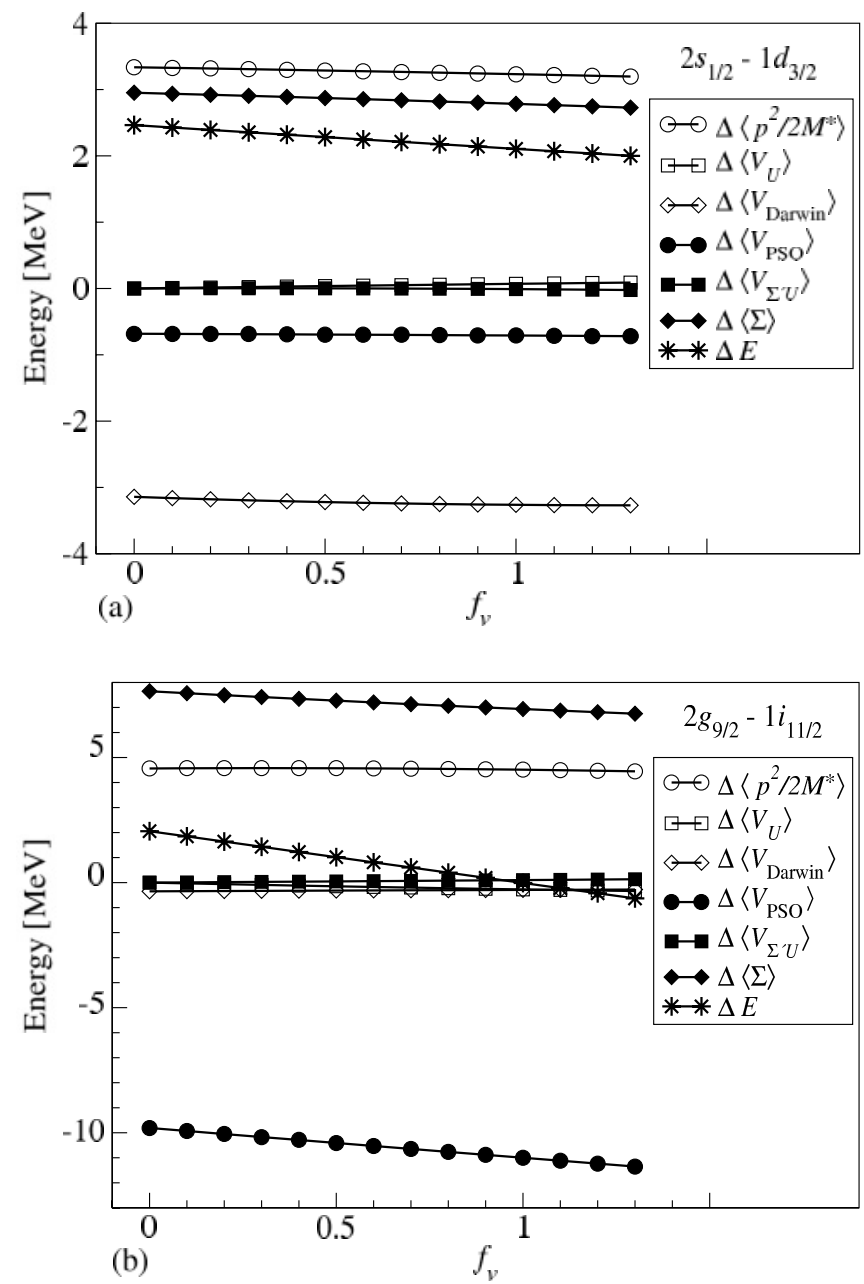

FIG. 2. The contributions from the terms of the energy decomposition [Eq. (23)] for pseudospin doublets (a) $\left[2 s_{1 / 2}-1 d_{3 / 2}\right]$ and (b) $\left[2 g_{9 / 2}-1 i_{11 / 2}\right]$ when $f_{v}$ varies from 0 to 1.3 .

potential $V_{\text {PSO }}$ almost does not change with $f_{v}$, whereas the contribution of terms such as $\left\langle p^{2} / 2 M^{*}\right\rangle$ and $\langle\Sigma\rangle$, which do not depend explicitly on the tensor potential $U$, is greater. This means that the main contribution to the change of pseudospin splitting with the strength of the tensor potential results mainly via the change of the wave function induced by $U$. Furthermore, the energy-splitting results for the most part from a cancellation of the $\Delta\langle\Sigma\rangle$ and $\Delta\left\langle V_{\text {Darwin }}\right\rangle$ contributions and also from $\Delta\left\langle p^{2} / 2 M^{*}\right\rangle$, the contribution from $V_{\mathrm{PSO}}$ having a lesser role. This agrees with previous findings of similar studies of pseudospin splittings $[15,16]$.

As far as the upper doublet $\left[2 g_{9 / 2}-1 i_{11 / 2}\right]$ is concerned, much of the previous analysis still holds, except for the fact that the pseudospin-orbit potential is much stronger and also changes sensibly as $f_{v}$ changes, being responsible for most of the pseudospin splitting. The reason for this, and in particular the fact that for values of $f_{v}$ greater than 0.95 the splitting becomes negative, that is, there is a level inversion, must be found in a more detailed analysis of the contribution of the tensor potential $U$ for the $V_{\text {Pso }}$ potential. From Eq. (24) we see that, whereas $U$ gives a positive contribution to this potential 

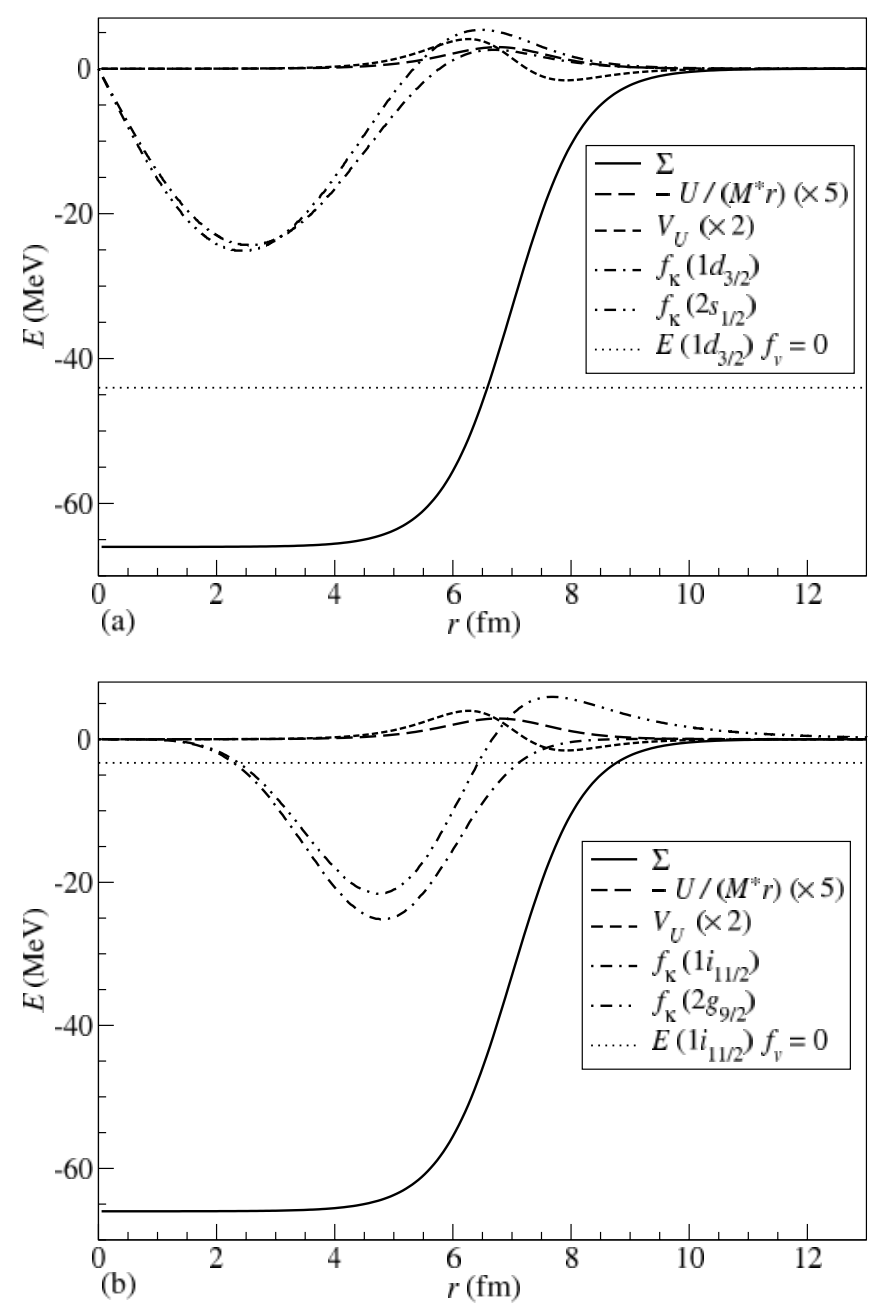

FIG. 3. The potentials $\Sigma, V_{U}$, and $-U /\left(M^{*} r\right)$ and the lower radial wave functions $f_{\kappa}$ for the pseudospin partners (a) $\left[2 s_{1 / 2}-1 d_{3 / 2}\right]$ and (b) $\left[2 g_{9 / 2}-1 i_{11 / 2}\right]$ when $f_{v}=1.2$. The wave functions were normalized such that they could be plotted side by side with the potentials.

[because $V^{\prime}<0$, so that, from Eq. (5), $-U>0$ ], the effective contribution depends on the sign of $\Sigma^{\prime} /(E-\Sigma) \times \boldsymbol{L} \cdot \boldsymbol{S} / r$. Because it changes sign when $r>r_{s}$, in which $r_{s}$ is the radius for which $\Sigma=E$, the net change of $\left\langle V_{\mathrm{PSO}}\right\rangle$ depends on how the wave function $f_{\kappa}(r)$ behaves near $r_{s}$ for both pseudospin partners. What we found is that for these surface levels, the following $U$ contribution for the pseudospin-orbit potential:

$$
V_{\mathrm{PSO}}^{U}=-\frac{2}{M^{*}} U \frac{\boldsymbol{L} \cdot \boldsymbol{S}}{r}
$$

is the dominant one. The reason why the contribution of this potential to $\Delta V_{\mathrm{PSO}}$ for the pair $\left[2 g_{9 / 2}-1 i_{11 / 2}\right]$ is so big lies in the fact that it is proportional to $\langle 2 \boldsymbol{L} \cdot \boldsymbol{S}\rangle=-(1-\kappa)$, which is equal to -6 and 5 for $2 g_{9 / 2}$ and $1 i_{11 / 2}$ respectively. Because $U$ is negative, $\left\langle V_{\mathrm{PSO}}^{U}\right\rangle\left(2 g_{9 / 2}\right)-\left\langle V_{\mathrm{PSO}}^{U}\right\rangle\left(1 i_{11 / 2}\right)$ is negative and more so as $f_{v}$ increases [see Fig. 2(b)], thus explaining why pseudospin splitting decreases with the increase of $f_{v}$.

Note that, from Table I, one is able to see directly the different effects that the tensor coupling has on $\Delta\left\langle V_{\mathrm{PSO}}\right\rangle$ in low-lying $\left(\left[2 p_{3 / 2}-1 f_{5 / 2}\right]\right)$ and surface $\left(\left[3 p_{3 / 2}-2 f_{5 / 2}\right]\right)$ pseudospin partners that have the same $\kappa$ values. The values of $\left\langle V_{\mathrm{PSO}}\right\rangle$ and their differences change significantly with $f_{v}$ for the surface partner.

Figs. 3(a) and (b) show $\Sigma, V_{U}$, the radial part of the $V_{\mathrm{PSO}}^{U}$ potential [Eq. (27)], given by $-U /\left(M^{*} r\right)$, which is always positive, as discussed, and the lower radial wave functions $f_{\kappa}$ for the pseudospin partners $\left[2 s_{1 / 2}-1 d_{3 / 2}\right]$ and $\left[2 g_{9 / 2}-\right.$ $\left.1 i_{11 / 2}\right]$, respectively, when $f_{v}=1.2$. Also plotted is the energy of the lowest lying level of each doublet for $f_{v}=0$, allowing to have a rough estimate of the value $r_{s}$ mentioned above by the intersection of its horizontal line with the $\Sigma$ potential curve. From these figures one sees clearly why the tensor potential $U$ has a much larger effect for a surface pseudospin doublet. Indeed, the lower radial wave functions for these levels have a significant strength near the nucleus surface, such that $\left\langle V_{\mathrm{PSO}}^{U}\right\rangle$ can have a significant value and have a sizeable contribution to the pseudospin splitting.

\section{CONCLUSIONS}

In this article we assessed the importance of the isoscalar tensor coupling to pseudospin symmetry as is realized in heavy nuclei, using as an example the neutron pseudospin partners of ${ }^{208} \mathrm{~Pb}$, calculated within a relativistic theory with scalar and vector mean fields parametrized with Woods-Saxon potential forms. By looking into the second-order equation for the lower component of the Dirac spinor, we obtained an expression for the pseudospin-orbit potential, showing its explicit dependence on the radial tensor potential. Because this potential comes from a derivative coupling (higher order term in the Lagrangian), the contribution for the energy from the potentials originated by that interaction are all scaled by $1 / M$ ( $M$ is the nucleon mass) and, because of that, is very small in comparison with kinetic and potential terms. However, because the spin-orbit interaction (and pseudospin) is a term of the same order, the effect of the tensor coupling can be significant in this case. In fact, we conclude that the contribution from $U$ to the pseudospin potential $V_{\mathrm{PSO}}$ is the dominant one for the surface levels.

We have shown that the surface pseudospin partners were the most affected by the tensor coupling, as was expected, and that this coupling reduces pseudospin splitting. This reduction can be significative to the point of inverting the level order, with the states with aligned spin $(j=l+1 / 2)$ having higher energy than the states with antialigned spin.

By analyzing the several contributions to the pseudospin energy splittings, we were able to confirm a conclusion reached in previous works, namely that the pseudospin symmetry is realized dynamically in nuclei, resulting from a cancellation of the various contributions rather than just the one from the pseudospin-orbit potential. We also found a systematic change in the energy for the pseudospin partners when we increase the tensor coupling $f_{v}$ : states with aligned spin $(\kappa<0)$ become deeper in opposite to antialigned ones that become more unbounded. This systematics allows us to conclude that pseudospin symmetry is improved by the tensor interaction. Finally, we were able to show for a surface pseudospin doublet the pseudospin-orbit potential, especially through its tensor 
potential part, gives a significant contribution to the change of the energy splitting. This finding is compatible with the dynamical character of the pseudospin symmetry.

\section{ACKNOWLEDGMENTS}

We acknowledge financial support from CNPq, FAPESP, and FCT (POCTI) scientific program. R.L. and M.M. acknowledge, in particular, the $\mathrm{CNPq}$ support and A.S.C was also supported by FAPESP.

\section{APPENDIX} (12).

In this Appendix we present the derivation of Eqs. (11) and

If we define the operator $\mathcal{O}=\boldsymbol{\alpha} \cdot \boldsymbol{p}+i \boldsymbol{\alpha} \cdot \hat{\boldsymbol{r}} U$, the secondorder differential equations are obtained by applying $\mathcal{O O}^{\dagger}$ and $\mathcal{O}^{\dagger} \mathcal{O}$ to $\Psi_{-}$and $\Psi_{+}$, respectively. For $\Psi_{-}$we have the following:

$$
\begin{aligned}
\mathcal{O O}^{\dagger} \Psi_{-} & =\mathcal{O}(E-\Sigma) \Psi_{+} \\
& =[\boldsymbol{\alpha} \cdot \boldsymbol{p}(E-\Sigma)] \Psi_{+}+(E-\Sigma) \mathcal{O} \Psi_{+} \\
& =i \boldsymbol{\alpha} \cdot \hat{\boldsymbol{r}} \Sigma^{\prime} \Psi_{+}+(E-\Sigma)(E+2 M-\Delta) \Psi_{-} \\
& =\left(\boldsymbol{p}^{2}+U^{2}-U^{\prime}-2 \frac{U}{r}-4 U \frac{\boldsymbol{L} \cdot \boldsymbol{S}}{r}\right) \Psi_{-},
\end{aligned}
$$

where $\Sigma, \Delta$, and $U$ are radial potentials, primes denote derivatives with respect to $r$, and $S$ stands for the $4 \times 4$ spin matrix. Conversely, from the equation $\mathcal{O}^{\dagger} \Psi_{-}=(E-\Sigma) \Psi_{+}$ we obtain the following:

$$
\begin{aligned}
\mathcal{O}^{\dagger} \Psi_{-} & =(\boldsymbol{\alpha} \cdot \boldsymbol{p}-i \boldsymbol{\alpha} \cdot \hat{\boldsymbol{r}} U) \Psi_{-} \\
& =\boldsymbol{\alpha} \cdot \hat{\boldsymbol{r}}(\hat{\boldsymbol{r}} \cdot \boldsymbol{p}+i \hat{\boldsymbol{r}} \times \boldsymbol{p} \cdot \boldsymbol{\Sigma}-i U) \Psi_{-} \\
& =-i \boldsymbol{\alpha} \cdot \hat{\boldsymbol{r}}\left(\frac{\partial \Psi_{-}}{\partial r}+U \Psi_{-}-2 \frac{\boldsymbol{L} \cdot \boldsymbol{S}}{r} \Psi_{-}\right) \\
& =(E-\Sigma) \Psi_{+},
\end{aligned}
$$

which allows us to write the following:

$$
\begin{aligned}
i \boldsymbol{\alpha} \cdot \hat{\boldsymbol{r}} \Sigma^{\prime} \Psi_{+} & =i \boldsymbol{\alpha} \cdot \hat{\boldsymbol{r}} \Sigma^{\prime} \frac{\mathcal{O}^{\dagger} \Psi_{-}}{E-\Sigma} \\
& =\frac{\Sigma^{\prime}}{E-\Sigma}\left(\frac{\partial \Psi_{-}}{\partial r}+U \Psi_{-}-2 \frac{\boldsymbol{L} \cdot \boldsymbol{S}}{r} \Psi_{-}\right) .
\end{aligned}
$$

In the same way, for $\Psi_{+}$we have the following:

$$
\begin{aligned}
\mathcal{O}^{\dagger} \mathcal{O} \Psi_{+} & =i \boldsymbol{\alpha} \cdot \hat{\boldsymbol{r}} \Delta^{\prime} \Psi_{-}+(E-\Sigma)(E+2 M-\Delta) \Psi_{+} \\
& =\left(\boldsymbol{p}^{2}+U^{2}+U^{\prime}+2 \frac{U}{r}+4 U \frac{\boldsymbol{L} \cdot \boldsymbol{S}}{r}\right) \Psi_{+},
\end{aligned}
$$

and, using equation $\mathcal{O} \Psi_{+}=(E+2 M-\Delta) \Psi_{-}$, we get the following:

$$
\begin{aligned}
i \boldsymbol{\alpha} \cdot \hat{\boldsymbol{r}} \Delta^{\prime} \Psi_{-}= & \frac{\Delta^{\prime}}{E+2 M-\Delta} \\
& \times\left(\frac{\partial \Psi_{+}}{\partial r}-U \Psi_{+}-2 \frac{\boldsymbol{L} \cdot \boldsymbol{S}}{r} \Psi_{+}\right) .
\end{aligned}
$$

Using Eqs. (A1) and (A2) for $\Psi_{-}$and Eqs. (A3) and (A4) for $\Psi_{+}$, we get finally Eqs. (11) and (12).
[1] K. T. Hecht and A. Adler, Nucl. Phys. A137, 129 (1969).

[2] A. Arima, M. Harvey, and K. Shimizu, Phys. Lett. B30, 517 (1969).

[3] C. Bahri, J. P. Draayer, and S. A. Moszkowski, Phys. Rev. Lett. 68, 2133 (1992).

[4] A. L. Blokhin, C. Bahri, and J. P. Draayer, Phys. Rev. Lett. 74, 4149 (1995).

[5] J. Meng, K. Sugawara-Tanabe, S. Yamaji, P. Ring, and A. Arima, Phys. Rev. C 58, 628(R) (1998).

[6] J. Meng, K. Sugawara-Tanabe, S. Yamaji, and A. Arima, Phys. Rev. C 59, 154 (1999).

[7] J. N. Ginocchio, Phys. Rev. Lett. 78, 436 (1997); Phys. Rep. 315, 231 (1999).

[8] J. S. Bell and H. Ruegg, Nucl. Phys. B98, 151 (1975).

[9] J. D. Walecka, Ann. Phys. (NY) 83, 491 (1974).

[10] B. D. Serot and J. D. Walecka, in Advances in Nuclear Physics, edited by J. W. Negele and E. Vogt (Plenum, New York, 1986), Vol. 16.

[11] R. J. Furnstahl and B. D. Serot, Phys. Rev. C 41, 262 (1990).

[12] J. N. Ginocchio and A. Leviatan, Phys. Lett. B245, 1 (1998).

[13] J. N. Ginocchio and A. Leviatan, Phys. Rev. Lett. 87, 072502 (2001).

[14] P. Alberto, M. Fiolhais, M. Malheiro, A. Delfino, and M. Chiapparini, Phys. Rev. Lett. 86, 5015 (2001).
[15] P. Alberto, M. Fiolhais, M. Malheiro, A. Delfino, and M. Chiapparini, Phys. Rev. C 65, 034307 (2002).

[16] R. Lisboa, M. Malheiro, and P. Alberto, Phys. Rev. C 67, 054305 (2003).

[17] R. Lisboa, M. Malheiro, and P. Alberto, Braz. J. Phys. 34, 293 (2004).

[18] S. Marcos, L. N. Savushkin, M. López-Quelle, and P. Ring, Phys. Rev. C 62, 054309 (2000).

[19] S. Marcos, M. López-Quelle, R. Niembro, L. N. Savushkin, and P. Bernardos, Phys. Lett. B513, 30 (2001).

[20] R. J. Furnstahl, J. J. Rusnak, and B. D. Serot, Nucl. Phys. A632, 607 (1998).

[21] G. Mao, Phys. Rev. C 67, 044318 (2003).

[22] R. Lisboa, M. Malheiro, A. S. de Castro, P. Alberto, and M. Fiolhais, Phys. Rev. C 69, 024319 (2004).

[23] J. N. Ginocchio, Phys. Rev. C 69, 034318 (2004).

[24] R. Lisboa, M. Malheiro, A. S. de Castro, P. Alberto, and F. Fiolhais, Int. J. Mod. Phys. D 13, 1447 (2004).

[25] M. Chiapparini, A. O. Gatone, and B. K. Jennings, Nucl. Phys. A529, 589 (1991).

[26] R. J. Furnstahl, B. D. Serot, and H.-B. Tang, Nucl. Phys. A615, 441 (1997).

[27] X. Campi and D. W. Sprung, Nucl. Phys. A399, 529 (1983). 